\title{
Effet des coûts de transaction sur la performance économique et l'adoption du coton biologique au Centre et Nord du Bénin
}

\author{
Barnabé AGALATI $^{*}$ et Pamphile DEGLA ${ }^{1,2}$ \\ ${ }^{1}$ Laboratoire d'Analyses et de Recherches sur les Dynamiques Economiques et Sociales (LARDES / FA / UP). \\ ${ }^{2}$ Département d'Economie et de Sociologie Rurales, Faculté d'Agronomie, Université de Parakou. \\ BP : 123 Parakou / Benin. \\ *Auteur correspondant ; E-mail: agalatibarnab88@gmail.com; Tel : +22997401385/94052517
}

\begin{abstract}
RESUME
Face au défi de la dégradation de l'environnement et des problèmes sanitaires liés à la production du coton conventionnel au Bénin, la production du coton biologique initiée depuis quelques décennies peine à se développer. Cet article s'intéresse à l'analyse de l'effet des coûts de transaction (CT) sur la performance économique et l'adoption du coton biologique au Centre et au Nord du Bénin. Basée sur un échantillon aléatoire de 408 producteurs dont 168 adoptants du coton biologique, l'étude a utilisé l'approche d'estimation des CT, la régression logistique et le test $t$ de Student pour l'analyse des données. Les résultats montrent que les CT, plus élevés dans le système du coton biologique réduisent considérablement la performance économique de ce système et affectent négativement la probabilité de son adoption. Outre cet effet, il ressort également l'influence négative d'autres facteurs tels que le sexe, le niveau de rendement, la distance domicile-exploitation, le nombre d'années d'expérience dans la production cotonnière et le mode de faire valoir direct sur l'adoption du coton biologique. La formation technique dans la production du coton biologique et la situation géographique exercent par contre une influence positive sur l'adoption du coton biologique.

(C) 2020 International Formulae Group. All rights reserved.
\end{abstract}

Mots clés : Déterminants, système de production, économie néo-institutionnelle, agriculture biologique

\section{Effect of transaction costs on the economic performance and the adoption of organic cotton in central and northern Benin}

\begin{abstract}
Regarding the environmental degradation challenge and health problems due to the production of conventional cotton in Benin, organic cotton production initiated several decades ago is struggling to develop. This paper focuses on analyzing the effect of transaction costs on the economic performance and the adoption of organic cotton in central and northern Benin. The study is based on a random sample of 408 producers, including 168 adopters of organic cotton. The transaction costs estimation approach, the logistic regression and the Student's t-test were used for data analysis. The results show that the high transaction costs in the organic cotton system significantly reduce the economic performance of this system and negatively affect the probability of adoption of organic cotton. In addition, there is the negative influence of other factors such as gender, the level of yield, the distance from home to farm, the years of experience in cotton production as well as the direct tenure mode in the adoption of organic cotton. On the other side, technical training in the production of organic cotton and the geographic location have a positive influence on the adoption of organic cotton.
\end{abstract}

(C) 2020 International Formulae Group. All rights reserved.

Keywords: Determinants, production system, new institutional economics, organic production. 


\section{INTRODUCTION}

De par sa contribution en termes de valeur ajoutée (13\% du PIB), de valeur totale des exportations $(70 \%)$ et de rentrées fiscales hors douanes (35\%) en 2018 (INSAE, 2019), la filière cotonnière constitue la base de l'économie au Bénin. A ce titre, le coton occupe dans la politique agricole une place de choix faisant de lui la filière la plus subventionnée et la plus organisée de toutes les filières agricoles. Fort de cet appui, la production a connu ses dernières années notamment lors de la campagne 2017-2018 une évolution exponentielle atteignant même une croissance de $122 \%$ en volume et une augmentation de $74 \%$ des superficies emblavées au cours des deux dernières saisons (Vidjingninou, 2018). La quantité d'insecticide utilisée s'est aussi accrue de 158\%, montrant ainsi l'étroitesse du lien entre le niveau de production et la consommation en produits de traitement. En effet à elle seule, la culture du coton consomme près de $96 \%$ des engrais chimiques et $90 \%$ des insecticides utilisés chaque année dans le secteur agricole au Bénin (Ton, 2001 ; Gouda et al., 2018). S'il est vrai que ces produits phytosanitaires contribuent à l'amélioration de la productivité, de nombreux problèmes sanitaires et environnementaux observés dans la zone cotonnière sont cependant attribuables à l'utilisation de ces produits. Et ceci, du fait de l'utilisation excessive et mal maitrisée de la plupart de ces produits phytosanitaires dont l'origine et la qualité sont souvent douteuses (Agbohessi et al. 2012). Outre ces problèmes sanitaires et environnementaux, la dégradation des sols en termes de perte de fertilité est évidente dans les zones cotonnières du Bénin du fait de l'impact sur la minéralisation de ces sols (Amonmidé et al. 2019).

Face à ces problèmes, la production du coton biologique a été introduite dans les zones cotonnières au centre et au nord du Bénin du fait de son rôle non polluant. Mais malgré ses avantages comparatifs en termes économique (Tovignan et al., 2018), sanitaire et environnemental (Sodjinou et al., 2015), la production biologique peine à se développer du fait d'un certain nombre de contraintes freinant l'essor de cette culture. La pénibilité du travail, l'indisponibilité des intrants biologiques et le coût de la procédure de certification sont considérés comme étant des facteurs liés à la faible diffusion du coton biologique (Houndékon, 2014 ; Bonou-Zin, 2012 ; Hountondji, 2015). Pour d'autres auteurs, le niveau d'instruction, le faible revenu des producteurs et leur situation géographique influencent aussi négativement l'adoption du coton biologique (Sodjinou et al., 2015). Outre ces facteurs il y a cependant d'autres contraintes majeures telles que les coûts de transaction (CT), mais non abordés jusqu'ici dans aucune de ces études et dont l'influence pourrait être non négligeable sur la diffusion de la production biologique. En effet, l'organisation de la production et la mise en marché du coton biologique génèrent des coûts d'échange liés à la recherche d'information et de prise de contact avec des partenaires à tous les niveaux du processus de production et de vente dont l'ampleur pourrait être importante du fait surtout de l'asymétrie informationnelle entre les différents acteurs. Selon l'approche néo-institutionnelle, de tels coûts longtemps négligés dans l'analyse économique classique sont pourtant des coûts réels dont l'ampleur affecte la performance économique de tout système de production ou d'échange (Degla, 2017). Face à de tels coûts, les agents économiques choisissent le système économique offrant le meilleur avantage comparatif en termes de réduction de coûts de transaction (Slangen et al., 2008; Degla, 2017). Ainsi dans le choix du système de production $\mathrm{du}$ coton conventionnel ou biologique, l'ampleur des coûts de transaction pourrait être déterminante. En s'intéressant à l'analyse de ces coûts, la présente étude vise non seulement à contribuer à l'opérationnalisation des coûts de transaction dans la littérature économique, mais aussi, mettre en évidence une contrainte majeure, jusqu'ici négligée dans l'analyse du processus de diffusion du coton biologique au Bénin et dont la prise en compte par les décideurs de politique agricole pourrait favoriser 
l'expansion de ce système cotonnier réducteur d'externalités négatives.

\section{MATERIEL ET METHODES \\ Coûts de transaction et adoption d'innovation}

Si l'origine des coûts de transaction (CT) est attribuable à Coase (1937), c'est cependant l'émergence du courant de pensée néo-institutionnelle à travers surtout les travaux de Stiglitz $(1986,1988)$ et de Williamson (1990, 1994, 1998, 2000) qui a donné un regain d'intérêt à ce concept. Selon l'angle d'analyse, le concept de CT peut prendre diverses connotations allant des coûts d'investissement (Klaes, 2000), ou coûts de fonctionnement (Arrow, 1969), coûts administratifs (Stiglitz, 1988) aux coûts d'échange (Benham et Benham, 2005). Mais d'un point de vue néo-institutionnel, les CT désignent les coûts liés à la recherche et la collecte d'information, la négociation, la signature et à l'exécution de contrats dans un système d'échange. Ces différents coûts liés au fonctionnement du marché et qui ne doivent pas être assimilés aux coûts de production (Degla, 2012) sont des coûts réels pouvant influencer l'efficacité de tout système économique (Eggertson, 1990). L'unité d'analyse dans l'approche des coûts de transaction étant la transaction, la théorie des CT essaie d'expliquer quel arrangement institutionnel ou mode d'organisation a l'avantage comparatif en cas de transactions (Slangen et al., 2008). Dans la présente étude, les CT sont considérés comme étant les coûts d'organisation de la production et d'échange du coton sur le marché dont, l'ampleur peut être décisive dans l'adoption ou non du système de production biologique. La culture biologique étant considérée comme une innovation agricole par rapport au système conventionnel, le processus d'adoption de telles innovations est largement abordé dans la littérature. A cet effet, le modèle de diffusion des innovations le plus souvent utilisé mais aussi souvent critiqué est celui de Rogers (1962, 1983). Ce modèle définit les éléments agissant sur la rapidité avec laquelle une innovation est adoptée dans un groupe social (Deschesnes et al., 2008). L'un des éléments clés est la persuasion qui est la phase où l'individu amorce une prise de position au sujet de l'innovation, en réagissant en fonction des caractéristiques de l'innovation dont notamment son avantage relatif en termes économique et social. Bien qu'étant critiqué comme étant un processus plutôt linéaire et mécaniste, ce modèle sert d'ancrage théorique pour la présente étude.

\section{Zone d'étude et collecte de données}

L'étude a été réalisée dans les trois Pôles de Développement Agricole (PDA) considérés comme les grands pôles cotonniers du Bénin (PDA 2, PDA 3 et PDA 4). Des communes abritant ces pôles, celles de Banikoara, Kandi, Tanguiéta et de Glazoué ont été choisies en raison de leur importance dans la production cotonnière (Figure 1). Dans chacune des communes, deux villages produisant simultanément les deux types de coton ont été retenus. Dans chacun des villages, 60 producteurs du coton conventionnel et $42 \mathrm{du}$ coton biologique ont été sélectionnés au hasard à partir d'une liste des producteurs fournie par le service de vulgarisation agricole. Ainsi, un échantillon de 408 producteurs dont 168 adoptants du coton biologique et 240 produisant le coton conventionnel a été constitué. Toutefois, le choix a tenu compte de façon égale des catégories de producteurs (grands producteurs, producteurs moyens et petits producteurs) au niveau de chaque système de production et village. L'effectif retenu pour chaque système de production du coton se justifie par le souci de prendre en compte dans l'échantillon les différentes catégories de producteurs du coton, de la connaissance du coton biologique par tous les producteurs de l'échantillon, de l'ancienneté des producteurs du coton biologique (au moins trois ans dans la pratique du coton biologique) et la disponibilité des producteurs à se prêter à nos questions. La collecte des données s'est faite sur la base d'entretiens individuels semistructurés, de focus groupes et de la technique de triangulation. Les données primaires 
collectées se rapportent aux caractéristiques socioéconomiques des producteurs, aux systèmes de production, aux diverses charges et coûts d'opportunité et autres coûts. Quant aux données secondaires, elles concernent les informations générales sur la production cotonnière et ont été collectées dans diverses sources de documentation. L'analyse des données a été faite grâce au logiciel Stata 15.

\section{Méthode d'estimation des coûts de transaction}

Deux méthodes d'estimation des CT sont couramment utilisées: la méthode indirecte et la méthode directe. Pendant que la première ne cherche pas à quantifier les $\mathrm{CT}$, la méthode directe retenue pour cette étude est basée sur la quantification. Généralement, les CT sont répartis en coûts avant (ex ante) et après (ex post) la signature du contrat. Dans le cas de notre étude, l'organisation de la production représente la phase ex ante de tout le processus et la mise en marché du coton graine la phase ex post. Ainsi, les composantes des coûts ex ante sont consignés dans le Tableau 1.

De la même manière, les coûts ex-post sont ceux liés à la mise en marché du coton graine dont les différentes composantes sont résumées dans le Tableau 2.

Dans la présente étude, l'estimation des CT s'appuie sur le modèle de Degla (2012) qui est basé non seulement sur le concept de coûts d'opportunité de Benham et Benham (2005) mais aussi sur le concept de «Non-market transaction costs » de North (1987) et De Soton (1990). Ainsi, dans chacune des deux phases ex ante et ex post précédemment définies le modèle est appliqué comme suit :

$C T 1_{K}=\sum_{I=1}^{N} w T_{I K}+C_{I K}$

Avec w le taux de salaire dans la zone d'étude, $\mathrm{T}_{\mathrm{ik}}$ le temps consacré par le producteur i pour une opération donnée (organisation de la production) dans le mode de production $\mathrm{k}, \mathrm{N}$ le nombre total de producteurs, $\mathrm{C}_{\mathrm{Ik}}$ les charges monétaires engagés au cours des activités (communication, déplacement, etc.) par le producteur i dans le mode k et $C T 1_{K}$ les coûts de transaction ex ante pour le mode $\mathrm{k}$.

Par analogie les coûts de transaction $e x$ post (CT2) peuvent s'exprimer également comme suit :

$C T 2_{K}=\sum_{I=1}^{N} w T_{I K}+D_{I K}$

Ainsi, le coût de transaction total généré au niveau de chaque système de production devient :

$C T_{k}=C T 1_{k}+C T 2_{k}$

Les CT ont été estimés en FCFA par hectare. Dans l'estimation des indicateurs de performance économique de chacun des systèmes de production, les CT ont été pris en compte c'est-à-dire internalisés. Ensuite, la comparaison des différents résultats des deux systèmes de production de coton a été faite à l'aide du test $t$ de Student.

\section{Spécification du modèle de régression}

Dans l'étude d'adoption d'innovation, plusieurs modèles économétriques sont généralement utilisés dont les plus courants sont les modèles Logit et probit (Verbeek, 2004 ; Greene, 2008). Mais en dépit des différences caractéristiques des deux modèles, ils conduisent selon Green (2008) souvent à la même conclusion et ainsi il est difficile de faire un choix entre les deux modèles sur des bases théoriques. Dans la présente étude, le modèle Logit a été retenu à cause de sa souplesse. La variable dépendante étant le statut d'adoption du producteur, avec 1 si le coton biologique est adopté et 0 si c'est le coton conventionnel. Selon Yabi et al. (2016), la forme fonctionnelle d'un tel modèle peut être représentée par l'équation empirique suivante :

$$
P(Y i=1 / b i o)=\frac{1}{1+e^{-X}}
$$

(4) avec

$X=\beta_{0}+\beta_{1} \ln (C T)+\beta_{2}$ Kandi +

$\beta_{3}$ (Glazoué) $+\beta_{4}(\operatorname{Mod})+$

$\beta_{5} \ln ($ DISTEX $)+\beta_{6} \ln ($ EXPCOT $)+$ 
$\beta_{7}($ Formabio $)+\beta_{8} \ln ($ Rend $)+\beta_{9}$ Sex $+e_{j}$

(5)

Où $\beta_{0}$ le terme constant, $\beta_{j}$ les coefficients à estimer, et $e_{j}$ les termes d'erreur. La qualité de ce modèle a été appréciée avec la méthode du maximum de vraisemblance qui suit la loi de khi deux. Le choix des variables explicatives introduites dans le modèle et décrites dans le Tableau 3, a été effectué sur la base d'une revue de littérature et des observations faites sur le terrain.

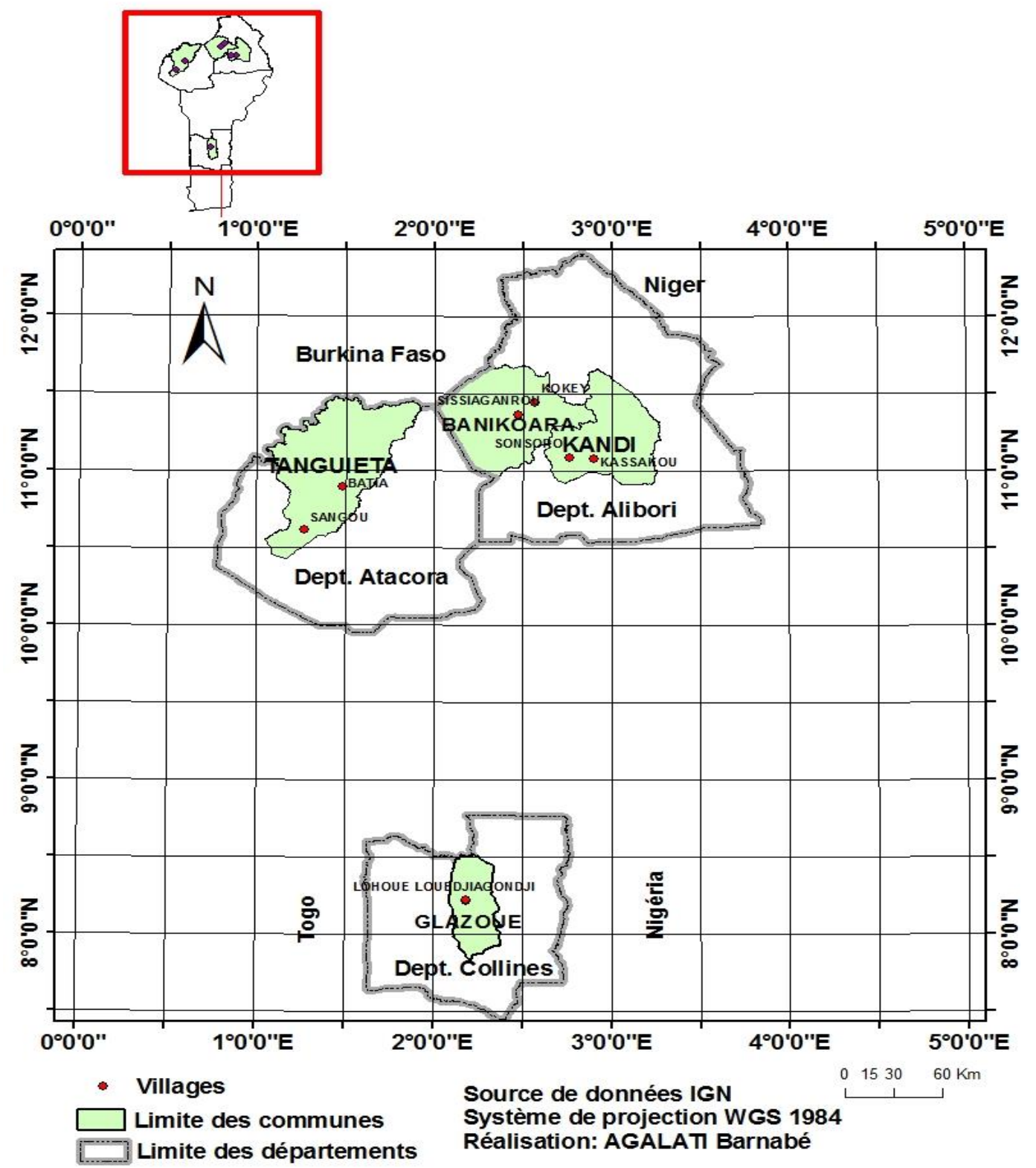

Figure 1 : Carte de localisation de la zone d'étude. 
Tableau 1 : Composantes des coûts de transaction ex-ante.

\begin{tabular}{|c|c|c|}
\hline Activités & $\begin{array}{l}\text { Type de coût de } \\
\text { transaction }\end{array}$ & Eléments de coût de transaction $e x$-ante \\
\hline $\begin{array}{l}\text { Organisation de } \\
\text { la production du } \\
\text { coton }\end{array}$ & $\begin{array}{l}\text { Coût de } \\
\text { transaction } e x- \\
\text { ante } \\
\text { (CT1) }\end{array}$ & $\begin{array}{l}\text { - Part individuelle du coût d'opportunité collectif du : } \\
\text {-Temps de développement de stratégies de renforcement } \\
\text { éventuel de l'accord entre producteurs et les coopératives } \\
\text { pour la fourniture d'intrants ; } \\
\text {-Temps de recherche d'information par la coopérative sur les } \\
\text { intrants coton (qualité, quantité, disponibilité, période de } \\
\text { convoi) via les appels téléphoniques aux agents de la société } \\
\text { de développement du coton (SODECO); } \\
\text {-Temps de négociation avec le transporteur des intrants ; } \\
\text {-Temps de signature du contrat de fourniture de intrants ; } \\
\text {-Temps d'organisation des réunions avec les membres du } \\
\text { comité communal de suivi de la production du coton; } \\
\text {-Temps d'organisation des réunions de partage d'intrants } \\
\text { avec les producteurs ; } \\
\text {-Temps d'organisation du transport, des chargements, } \\
\text { déchargements d'intrants coton } \\
\text {-Temps de règlements d'éventuels conflits portant sur les } \\
\text { intrants entre les membres de la coopérative. } \\
\text { - Part individuelle des frais collectifs de : } \\
\text {-Communication avec les différents acteurs des coopératives } \\
\text { dans le cadre de distribution des intrants. } \\
\text { - Coût d'opportunité individuel du temps de : } \\
\text {-Recherche d'information par les producteurs sur les intrants } \\
\text { coton à échanger (qualité, quantité, disponibilité, prix et } \\
\text { conditions de vente); } \\
\text {-Recherche d'autres fournisseurs potentiels d'intrants coton } \\
\text { par les producteurs (localisation, contact, réputation) } \\
\text {-Participation aux réunions de la coopérative et relatives à } \\
\text { l'acquisition et la distribution d'intrants. } \\
\text { - Frais individuels de: } \\
\text {-Communication avec les secrétaires des coopératives et } \\
\text { agents de vulgarisation ; } \\
\text {-Communication avec d'éventuels nouveaux fournisseurs ; } \\
\text {-Déplacement vers les centres de réunions de distribution } \\
\text { d'intrants ; } \\
\text {-Déplacement éventuel vers de nouveaux fournisseurs } \\
\text { d'intrants ; } \\
\text {-Éventuelles autres dépenses monétaires (repas, etc.) lors des } \\
\text { réunions. }\end{array}$ \\
\hline
\end{tabular}


Tableau 2 : Composantes des coûts de transaction ex-post.

\begin{tabular}{|c|c|c|}
\hline Activités & $\begin{array}{l}\text { Type de coût de } \\
\text { transaction }\end{array}$ & Eléments de coût de transaction $e x$-post \\
\hline $\begin{array}{l}\text { Mise en } \\
\text { marché du } \\
\text { coton graine }\end{array}$ & $\begin{array}{l}\text { Coût de } \\
\text { transaction } e x- \\
\text { post } \\
\text { (CT2) }\end{array}$ & $\begin{array}{l}\text { • Part individuelle du coût d'opportunité collectif } \\
\text { du : } \\
\text {-Temps de développement d'une stratégie de négociation } \\
\text { avec l'acheteur, le contrôleur de la qualité et le transporteur } \\
\text { du coton graine; } \\
\text {-Temps de recherche d'information des coopératives sur le } \\
\text { coton graine à échanger (période de commercialisation et } \\
\text { prix); } \\
\text {-Temps de recherche d'information des coopératives sur les } \\
\text { transporteurs (état du camion, capacité, comportement } \\
\text { opportuniste du transporteur) du coton graine ; } \\
\text {-Temps d'organisation des réunions par les coopératives } \\
\text { pour de nettoyage des marchés autogérés et de } \\
\text { sensibilisation sur les bonnes pratiques de commercialisation } \\
\text { du coton graine; } \\
\text {-Temps de négociation avec le transporteur de coton graine } \\
\text { et des contrôleurs à l'usine; } \\
\text {-Temps de règlement d'éventuels conflits entre les acteurs } \\
\text { de la commercialisation du coton graine (producteurs, } \\
\text { coopératives, transporteurs, l'acheteur). } \\
\text { • Coût d'opportunité individuel du temps de : } \\
\text {-Recherche d'information individuelle sur le coton graine à } \\
\text { échanger (qualité, quantité, période de commercialisation et } \\
\text { prix) via les appels téléphoniques aux secrétaires des } \\
\text { coopératives et agents de vulgarisation ; } \\
\text {-Recherche d'autres acheteurs potentiels de coton graine } \\
\text { (localisation, contact, réputation) par les producteurs via les } \\
\text { déplacements et appels; } \\
\text {-Temps de participation aux réunions de partage de } \\
\text { nettoyage des marchés autogérés et de sensibilisation sur les } \\
\text { bonnes pratiques de commercialisation du coton graine ; } \\
\text {-Temps de surveillance du coton graine dans le marché } \\
\text { autogéré. } \\
\text { • Frais individuels de : } \\
\text {-Communication avec les secrétaires des coopératives et } \\
\text { autre agents de commercialisation } \\
\text {-Communication avec d'éventuels nouveaux acheteurs ; } \\
\text {-Déplacement vers les centres de réunions ; } \\
\text {-Déplacement éventuel vers de nouveaux acheteurs; } \\
\text {-Éventuelles autres dépenses monétaires lors des réunions. }\end{array}$ \\
\hline
\end{tabular}


Tableau 3 : Variables explicatives du modèle.

\begin{tabular}{|c|c|c|c|}
\hline Variables & Code & Modalités & Signes attendus \\
\hline Sexe & Sex & $\begin{array}{l}1=\text { Masculin } \\
0=\text { Féminin }\end{array}$ & - \\
\hline Zone Glazoué & Glazoué & $\begin{array}{c}\text { 1= Glazoué } \\
0=\text { Kandi, } \\
\text { Banikoara et } \\
\text { Tanguiéta }\end{array}$ & $+/-$ \\
\hline Zone Kandi & kandi & $\begin{array}{c}1=\text { Kandi } \\
0=\text { Glazoué, } \\
\text { Banikoara et } \\
\text { Tanguiéta }\end{array}$ & $+/-$ \\
\hline Coûts de transaction & CT & & - \\
\hline Distance entre domicile et exploitation & DISTEX & & $+/-$ \\
\hline Rendement de coton & Rend & & + \\
\hline $\begin{array}{l}\text { Mode de faire valoir direct (droit de } \\
\text { propriété) }\end{array}$ & Mod & $\begin{array}{l}1=\text { Oui } \\
0=\text { Non }\end{array}$ & $+/-$ \\
\hline $\begin{array}{l}\text { Expérience du producteur dans la } \\
\text { production cotonnière }\end{array}$ & EXPCOT & & + \\
\hline $\begin{array}{l}\text { Formation en production du coton } \\
\text { biologique }\end{array}$ & Formabio & $\begin{array}{l}1=\text { Oui } \\
0=\text { Non }\end{array}$ & + \\
\hline
\end{tabular}

\section{RESULTATS \\ Caractéristiques socioéconomiques des producteurs}

Dans la zone d'étude la plupart des producteurs enquêtés sont de sexe masculin (75\%). Au sein du groupe des producteurs du coton conventionnel, la proportion des hommes est de $94 \%$ contre $6 \%$ de femmes (Tableau 4). Par contre, la catégorie des producteurs biologiques regroupe $52 \%$ de femmes contre $48 \%$ d'hommes. La majorité des producteurs enquêtés est mariée (93\%), et cette tendance est la même chez les producteurs conventionnels que biologiques. L'âge moyen de tous les producteurs enquêtés est de 37 ans $( \pm 10,55)$ et la plupart ont un niveau d'instruction correspondant au primaire. Ces tendances sont les mêmes dans les deux groupes de producteurs. Le nombre moyen d'actifs chez les producteurs biologiques est de 4 actifs $( \pm 3)$ contre 5 actifs $( \pm 3)$ chez leurs homologues conventionnels. Les producteurs conventionnels ont emblavé en moyenne 4,40 ha $( \pm 3,79)$ contre 1,24 ha $( \pm 1,18)$ chez les producteurs biologiques avec un rendement moyen respectif de $1229,21 \mathrm{~kg} / \mathrm{ha}( \pm 290,23)$ et de $886,04 \mathrm{~kg} / \mathrm{ha} \quad( \pm 356,50)$. Parmi les producteurs enquêtés $92,2 \%$ ont accès direct à la terre, c'est à dire qu'ils exercent un droit de propriété sur leurs terres. Pendant que seulement $88,1 \%$ ont un accès direct à la terre au niveau des adoptants du coton biologique, ce sont plutôt $95 \%$ des producteurs conventionnels qui ont un accès direct à la terre. En moyenne les producteurs biologiques parcourent $4,743 \mathrm{~km}( \pm 7,571)$ entre leur domicile et leur exploitation pendant que les producteurs conventionnels se déplacent sur $6,488 \mathrm{~km}( \pm 7,614) \mathrm{km}$. Le nombre d'années d'expérience dans la production cotonnière est de 12 ans $( \pm 9)$ chez les producteurs biologiques et de 17 ans $( \pm 10)$ chez les conventionnels (Tableau 5).

\section{Niveaux de coûts de transaction et leur effet sur la performance économique}

L'organisation de la production et la mise en marché du coton biologique ont généré 
en moyenne $48047 \mathrm{~F} \mathrm{CFA} / \mathrm{ha}( \pm 158$ 485) de CT contre $25660 \mathrm{~F} \mathrm{CFA} / \mathrm{ha}( \pm 46544)$ pour le coton conventionnel. En prenant en compte ces CT, le coût total de production du coton biologique subit une augmentation de $32 \%$ contre $12 \%$ pour le coton conventionnel (Tableau 6). Il existe une différence significative entre le niveau des CT générés par le système de production biologique et celui du coton conventionnel. Cette différence est aussi significative entre les CT liés à la mise en marché du coton graine des deux types de coton, puisque dans la mise en marché du coton graine, les producteurs du coton biologique supportent presque le double de leurs homologues du coton conventionnel. Quant à l'organisation de la production du coton, la différence entre les CT reste toutefois non significative entre les deux systèmes de production, malgré le niveau légèrement élevé des CT au niveau du système de production biologique.

Par rapport à la performance économique des systèmes de production cotonnière, l'internalisation des CT dans l'analyse de la performance économique des deux systèmes de production montre que le coût total de production du coton biologique s'accroit de $32 \%$ contre $12 \%$ pour le coton conventionnel. Ainsi, l'internalisation des CT réduit la marge nette du coton biologique de
$39 \%$ contre $25 \%$ pour le coton conventionnel. De la même façon la productivité moyenne de la main d'œuvre familiale diminue de $28 \%$ pour le coton biologique et de $24 \%$ pour le coton conventionnel (Tableau 7).

\section{Déterminants de l'adoption du coton biologique}

Les résultats de la régression Logit binaire indiquent que le modèle est globalement significatif au seuil de $1 \%$ et par conséquent les coefficients estimés sont statistiquement valables (Chi2 460,09; $p=0,000)$. Les variations des variables explicatives introduites dans le modèle expliquent $83,22 \%$ des variations observées au niveau de l'adoption du coton biologique. Le modèle a identifié neuf facteurs influençant l'adoption $\mathrm{du}$ coton biologique par les producteurs. Il s'agit des coûts de transaction, du sexe du producteur, le rendement du coton, le nombre d'années d'expérience dans la production cotonnière, la distance domicile exploitation et le mode de faire valoir direct qui influencent négativement la probabilité d'adoption du coton biologique pendant que l'appartenance aux zones géographiques (Kandi et Glazoué) et la formation technique en production de coton biologique influencent positivement la probabilité d'adoption du coton biologique (Tableau 8).

Tableau 4 : Caractéristiques sociodémographiques des enquêtés.

\begin{tabular}{llllllll}
\hline Variables qualitatives & \multicolumn{2}{c}{ Coton conventionnel } & \multicolumn{2}{c}{ Coton biologique } & \multicolumn{2}{l}{ Ensemble } \\
\cline { 2 - 8 } & & Effectif & Fréquence & Effectif & Fréquence & Effectif & Fréquence \\
\hline $\begin{array}{l}\text { Sexe du } \\
\text { producteur }\end{array}$ & Masculin & 226 & 94 & 80 & 48 & 306 & 75 \\
& Féminin & 14 & 6 & 88 & 52 & 102 & 25 \\
\hline $\begin{array}{l}\text { Situation } \\
\text { matrimoniale }\end{array}$ & Marié & 224 & 93,3 & 157 & 93,5 & 381 & 93,4 \\
& Célibataire & 13 & 5,4 & 8 & 4,8 & 21 & 5,1 \\
& Divorcé & 2 & 0,8 & 0 & 0 & 2 & 0,5 \\
\cline { 2 - 8 } & Veuf & 1 & 0,5 & 3 & 1,7 & 4 & 1 \\
\hline $\begin{array}{l}\text { Mode d'accès à } \\
\text { la terre }\end{array}$ & Direct & 228 & 95 & 148 & 88,1 & 376 & 92,2 \\
\hline
\end{tabular}


Tableau 5 : Caractéristiques socioéconomiques des enquêtés.

\begin{tabular}{lllllll}
\hline Variables quantitatives & \multicolumn{2}{l}{ Coton conventionnel } & \multicolumn{2}{l}{ Coton biologique } & \multicolumn{2}{l}{ Ensemble } \\
\cline { 2 - 7 } & Moyenne & Ecart type & Moyenne & Ecart type & Moyenne & Ecart type \\
\hline L'âge des producteurs & 37,18 & 10,55 & 37,49 & 10,76 & 37,31 & 10,56 \\
\hline Nombre d'actif agricole & 4,72 & 3,06 & 3,77 & 2,70 & 4,33 & 2,95 \\
\hline Superficie de coton & 4,40 & 3,79 & 1,24 & 1,18 & 3,10 & 3,39 \\
\hline Rendement & 1229,21 & 290,23 & 886,04 & 356,50 & 1087,91 & 360,84 \\
\hline $\begin{array}{l}\text { Distance domicile et } \\
\text { exploitation des producteurs }\end{array}$ & 6,49 & 7,61 & 4,74 & 7,57 & 5,78 & 7,64 \\
\hline $\begin{array}{l}\text { Nombre d'année d'expérience } \\
\text { dans la production cotonnière }\end{array}$ & 17,03 & 10,04 & 12,05 & 9,12 & 14,18 & 8,94 \\
\hline
\end{tabular}

Tableau 6: Coûts de transaction et de production du coton biologique et conventionnel.

\begin{tabular}{|c|c|c|c|}
\hline Catégorie de coûts de transaction & Système de production & Moyenne & Ecart-type \\
\hline \multirow{3}{*}{$\begin{array}{l}\text { Coûts de transaction liés à } \\
\text { l'organisation de la production du } \\
\text { coton (FCFA/ha) }\end{array}$} & Coton conventionnel & 9188 & 29165 \\
\hline & Coton biologique & 15882 & 112054 \\
\hline & Ensemble & 11944 & 75249 \\
\hline & \multicolumn{3}{|c|}{$\mathrm{t}=-0,884 ; \mathrm{ddl}=406 ; \mathrm{p}=0,337$} \\
\hline \multirow{4}{*}{$\begin{array}{l}\text { Coûts de transaction liés à la mise en } \\
\text { marché du coton graine (FCFA/ha) }\end{array}$} & Coton conventionnel & 16472 & 25888 \\
\hline & Coton biologique & 32165 & 60934 \\
\hline & Ensemble & 22934 & 44462 \\
\hline & \multicolumn{3}{|c|}{$\mathrm{t}=-3,558 ; \mathrm{ddl}=406 ; \mathrm{p}<0,001$} \\
\hline \multirow[t]{4}{*}{ Coût total de transaction (FCFA/ha) } & Coton conventionnel & 25660 & 46544 \\
\hline & Coton biologique & 48047 & 158485 \\
\hline & Ensemble & 34878 & 108166 \\
\hline & \multicolumn{3}{|c|}{$t=-2,066 ; d d l=406 ; p=0,039$} \\
\hline \multirow[t]{3}{*}{ Coût total de production biologique } & Sans CT & 151915 & 130908 \\
\hline & Avec CT & 199962 & 233652 \\
\hline & Variation & $32 \%$ & \\
\hline \multirow[t]{3}{*}{$\begin{array}{l}\text { Coût total de production } \\
\text { conventionnelle }\end{array}$} & Sans CT & 218780 & 807418 \\
\hline & Avec CT & 244440 & 807852 \\
\hline & Variation & $12 \%$ & \\
\hline
\end{tabular}


Tableau 7 : Performance économique des systèmes de production du coton avec ou sans les coûts de transaction.

\begin{tabular}{|c|c|c|c|c|c|c|}
\hline & \multicolumn{3}{|c|}{ Coton biologique } & \multicolumn{3}{|c|}{ Coton conventionnel } \\
\hline & Sans CT & Avec CT & Réduction & Sans CT & Avec CT & Réduction \\
\hline $\begin{array}{l}\text { Produit Brut valeur } \\
\text { (FCFA/Ha) }\end{array}$ & $\begin{array}{c}276604 \\
(111432)\end{array}$ & $\begin{array}{c}276604 \\
(111432)\end{array}$ & - & $\begin{array}{l}321759 \\
(86011)\end{array}$ & $\begin{array}{l}321759 \\
(86011)\end{array}$ & - \\
\hline \multirow{2}{*}{$\begin{array}{l}\text { Coûts de production } \\
\text { (FCFA/Ha) }\end{array}$} & $\begin{array}{c}151915 \\
(130908) \\
\end{array}$ & $\begin{array}{r}199962 \\
(233652) \\
\end{array}$ & - & $\begin{array}{l}218780 \\
(807418) \\
\end{array}$ & $\begin{array}{c}244440 \\
(807852) \\
\end{array}$ & - \\
\hline & \multicolumn{2}{|c|}{$\begin{array}{c}\mathrm{ddl}=167 ; \mathrm{t}=1,063 ; \mathrm{p} \\
\text { value }=0,288\end{array}$} & \multicolumn{4}{|c|}{$\mathrm{ddl}=239 ; \mathrm{t}=0,692 ; \mathrm{p}$-value $=0,489$} \\
\hline \multirow[t]{2}{*}{$\begin{array}{l}\text { Marge nette } \\
\text { (FCFA/Ha) }\end{array}$} & $\begin{array}{c}124690 \\
(158690) \\
\end{array}$ & $\begin{array}{c}76642 \\
(247818) \\
\end{array}$ & $39 \%$ & $102979(814890)$ & $\begin{array}{r}77319 \\
(815175) \\
\end{array}$ & $25 \%$ \\
\hline & \multicolumn{2}{|c|}{$\begin{array}{l}\mathrm{ddl}=167 ; \mathrm{t}=10,184 \\
\mathrm{p}=0,000\end{array}$} & & \multicolumn{2}{|c|}{$\mathrm{ddl}=239 ; \mathrm{t}=1,958 ; \mathrm{p}=0,051$} & \\
\hline \multirow[t]{2}{*}{$\begin{array}{l}\text { Productivité moyenne } \\
\text { de la main d'œuvre } \\
\text { (FCFA/homme-jour) }\end{array}$} & $\begin{array}{c}1830 \\
(3662)\end{array}$ & $\begin{array}{r}1326 \\
(3997)\end{array}$ & $28 \%$ & $\begin{array}{c}1819 \\
(10330)\end{array}$ & $\begin{array}{c}1387 \\
(10331)\end{array}$ & $24 \%$ \\
\hline & \multicolumn{2}{|c|}{$\begin{array}{c}\mathrm{ddl}=167 ; \mathrm{t}=6,477 ; \\
\mathrm{p}=0,000\end{array}$} & & \multicolumn{2}{|c|}{$\mathrm{ddl}=239 ; \mathrm{t}=2,727 ; \mathrm{p}=0,007$} & \\
\hline
\end{tabular}

Tableau 8 : Résultats du modèle d'adoption du coton biologique.

\begin{tabular}{|c|c|c|}
\hline Variables explicatives & Coefficients & Effets marginaux \\
\hline \multicolumn{3}{|l|}{ Sexe (Réf=Féminin) } \\
\hline Masculin & $-1,500 * *(0,734)$ & $-0,049 * *(0,023)$ \\
\hline \multicolumn{3}{|l|}{ Zone d'étude (Réf= Kandi, Banikoara, Tanguiéta) } \\
\hline Glazoué & $3,596 * * *(1,178)$ & $0,118 * * *(0,038)$ \\
\hline \multicolumn{3}{|l|}{ Zone d'étude (Réf= Glazoué, Banikoara, Tanguiéta) } \\
\hline Kandi & $4,446 * * *(1,127)$ & $0,146 * * *(0,034)$ \\
\hline Coûts de transaction & $-0,916^{* * *}(0,257)$ & $-0,030 * * *(0,008)$ \\
\hline Distance Domicile-exploitation & $-0,824 * * *(0,272)$ & $-0,027 * * *(0,008)$ \\
\hline Rendement du coton & $-3,020 * * *(0,592)$ & $-0,099 * * *(0,015)$ \\
\hline Formation en production du coton biologique & $9,634 * * *(1,488)$ & $0,315 * * *(0,035)$ \\
\hline $\begin{array}{l}\text { Nombre d'année d'expériences dans la production du } \\
\text { coton }\end{array}$ & $-1,250 * * *(0,439)$ & $-0,041 * * *(0,014)$ \\
\hline Mode de faire valoir direct & $-2,528 * * *(0,970)$ & $-0,083 * * *(0,030)$ \\
\hline Constante & $13,902 * * *(3,297)$ & --- \\
\hline Observations & \multicolumn{2}{|c|}{408} \\
\hline Log likelihood & \multicolumn{2}{|c|}{$-46,373$} \\
\hline R-square & \multicolumn{2}{|c|}{0,8322} \\
\hline Chi2 & \multicolumn{2}{|c|}{460,09} \\
\hline Probabilité & \multicolumn{2}{|c|}{0,0000} \\
\hline
\end{tabular}

( ) =Erreur standard ; ***; ** et * = significatif respectivement au seuil de 1\%, $5 \%$ et $10 \%$; c'est le logarithme de toutes les variables explicatives quantitative continue qui ont été introduit dans le modèle. 


\section{DISCUSSION}

Partant du rôle des coûts de transaction (CT) dans l'analyse théorique des choix des modes de gouvernance ou arrangements institutionnels, la présente étude a mis en évidence non seulement l'importance des CT dans les systèmes de production cotonnière au centre et au nord du Bénin mais aussi leur influence sur la performance économique et l'adoption du coton biologique. Il ressort des résultats qu'il y a une différence significative entre le niveau des CT générés par le système de production biologique (48 $047 \mathrm{FCFA} / \mathrm{ha}$ ) et celui du coton conventionnel (25660 FCFA/ha). Cette différence pourrait s'expliquer par le faible niveau d'organisation du système du coton biologique aussi bien dans la production que la commercialisation. L'internalisation des CT engendre une réduction des performances économiques des deux systèmes de production du coton mais de façon disproportionnée. Ainsi, ces résultats montrent que même si les deux systèmes de production cotonnière génèrent des $\mathrm{CT}$, c'est cependant le système du coton conventionnel qui offre le meilleur avantage comparatif en termes de réduction de ces $\mathrm{CT}$, ce qui pourrait aussi expliquer le manque d'engouement pour la production du coton biologique qui depuis plus de deux décennies peine à devenir une véritable alternative compétitive au coton conventionnel. Bien au contraire, l'ampleur relative des CT dans le système biologique influence négativement la probabilité d'adoption de ce système comme le montre l'analyse des déterminants de l'adoption du coton biologique. Ainsi, ces différents résultats confortent non seulement l'une des hypothèses de base de la théorie des CT stipulant le choix préférentiel du mode de gouvernance minimisant les CT par les agents économiques comme l'affirment Slangen et al. (2008) mais renforcent également les résultats empiriques de Dieye (2006) et Royer (2009) portant sur l'efficacité transactionnelle de différents modes de coordination de la mise en marché du lait respectivement au Québec et au Sénégal.

Outre l'influence des CT, les résultats montrent que lorsqu'on passe du sexe féminin au sexe masculin, la probabilité d'adoption du coton biologique diminue de $4,9 \%$, révélant ainsi le rôle prépondérant que jouent les femmes dans le développement du coton biologique dans la zone d'étude. Ce résultat corrobore ceux de Hougni et al. (2012) et Sodjinou et al. (2015) qui attestent aussi que les femmes sont plus favorables à la production du coton biologique que les hommes au Bénin. L'appartenance à la commune de Kandi dans la zone nord ou à celle de Glazoué dans la zone du centre augmente la probabilité d'adoption du coton biologique respectivement de 14,6\% et de $11,8 \%$. En effet, les deux zones font partie des premières communes d'introduction du coton biologique au Bénin. Ainsi, les producteurs de ces deux communes sont plus expérimentés et conscients des avantages comparatifs en termes économique et environnemental du coton biologique. Lequel n'est basé essentiellement que sur l'utilisation de la fumure organique par package des animaux ou par transport au champ pour améliorer la fertilité des sols. Et comme le confirment Bacyé et al. (2019) dans les zones cotonnières du Burkina Faso, le rôle de cette pratique de fertilisation incontournable dans l'amélioration de la qualité des sols. Quant au mode de faire valoir, lorsqu'on passe de la non jouissance de droit de propriété sur les terres à l'accès direct, la probabilité d'adoption du coton biologique diminue, suggérant que la plupart des propriétaires terrains préfèreraient s'adonner au coton conventionnel qui leur offre plus de garantie en termes d'accès aux intrants et de rendements plus élevés. En ce qui concerne la productivité du sol son influence négative sur l'adoption du coton biologique traduit le fait que la faiblesse des rendements inhérents au coton biologique (886 kg/ha contre $1229 \mathrm{~kg} / \mathrm{ha}$ pour le conventionnel) pousse les producteurs désireux d'avoir des 
rendements plus élevés vers le coton conventionnel. Cette faiblesse de rendement du coton biologique mise en évidence aussi par Azonkpin et al. (2018) dans leur étude sur la typologie des systèmes de production du coton biologique au Bénin et révélant que le système le plus performant n'atteint que $885 \mathrm{~kg} /$ ha reste à priori un handicap non négligeable dans l'adoption de ce type de coton par la plupart des adoptants potentiels. C'est ce que confirme aussi les observations similaires faites par Houndekon (2014) qui dans son étude attribue surtout la réticence des producteurs à l'adoption du coton biologique à notamment la faiblesse des rendements inhérents à cette culture. Ainsi, malgré la compensation qu'offrent les promoteurs du coton biologique à travers une majoration du prix d'achat du kilogramme de coton graine par rapport à celui $\mathrm{du}$ coton conventionnel, la faiblesse des rendements reste comme un frein psychologique que les promoteurs doivent essayer de corriger en s'investissant d'avantage dans la recherche d'intrants biologiques plus performants. Si la formation des producteurs dans la culture du coton biologique influence positivement la probabilité d'adoption du coton biologique, le nombre d'années d'expérience dans la production cotonnière et la distance domicile exploitation semblent par contre fidéliser les producteurs au système conventionnel dont ils maitrisent mieux les rouages comme le montre l'influence négative de ces deux facteurs sur la probabilité d'adoption du coton biologique.

\section{Conclusion}

Longtemps ignorés dans l'analyse économique classique, les CT constituent de nos jours des coûts réels qui sont l'expression monétaire d'un certain nombre de contraintes liées aux systèmes de production et d'échange. Ainsi, la présente étude s'est intéressée à l'analyse de l'effet des CT sur l'adoption du coton biologique, considérée comme un système de production alternatif non polluant au Bénin. Les résultats montrent que le niveau des CT résultant du système de coton biologique est plus important que celui du système conventionnel. L'internalisation des CT dans l'analyse économique des deux systèmes révèle un accroissement relativement plus important du coût total de production du coton biologique que celui du coton conventionnel. Cet accroissement a par conséquent induit une réduction de la marge nette et de la productivité de la main d'œuvre familiale fortement plus élevée au niveau du système biologique que conventionnel. Ainsi, en termes de minimisation des CT, la production biologique n'offre pas le meilleur avantage comparatif. Ceci pourrait expliquer la réticence des producteurs à adopter ce système comme le montre l'influence négative de l'ampleur des CT sur la probabilité d'adoption du coton biologique. Outre l'effet des CT, les résultats montrent également l'influence d'autres facteurs tels que la situation géographique, la formation technique en production du coton biologique, le sexe du producteur, le nombre d'années d'expérience dans la production cotonnière, le mode de faire valoir direct, la distance domicile exploitation et le rendement du coton sur l'adoption du coton biologique. Ainsi, pendant que la situation géographique et la formation technique en production du coton biologique influencent positivement la probabilité d'adoption du coton biologique, le sexe du producteur, le nombre d'années d'expérience dans la production cotonnière, le mode de faire valoir direct, la distance domicile exploitation et le rendement du coton exercent plutôt une influence négative sur la probabilité d'adoption. Au regard de ces résultats, il urge que l'Etat s'implique dans la promotion du coton biologique par une meilleure organisation des acteurs de la filière et la mise à disponibilité des intrants du coton biologique. Malgré la validité de ces résultats, une étude sur la perception des producteurs de l'importance de ces facteurs permettrait 
d'approfondir les connaissances sur l'influence réelle de ces facteurs, notamment les CT dont la prise en compte par les promoteurs de l'agriculture biologique devrait contribuer à assurer plus durablement la diffusion de la production du coton biologique. Une telle étude permettrait de mieux se focaliser sur cette contrainte majeure dont la levée ou du moins la réduction pourrait contribuer à assurer plus durablement l'essor de la production du coton biologique au Bénin.

\section{CONFLITS D'INTERETS}

Les auteurs déclarent qu'il n'y a aucun conflit d'intérêts.

\section{CONTRIBUTIONS DES AUTEURS}

Cet article est l'œuvre d'un auteur principal (BA) et d'un co-auteur (PD). BA est l'auteur qui a rédigé le protocole de recherche et conduit les enquêtes de terrain, conçu la base de données et analysé les données. C'est lui qui a proposé le premier draft de l'article, géré le processus de soumission et a suivi les recommandations des lecteurs sous la supervision du co-auteur PD. Par ailleurs, PD a contribué à la validation du protocole de recherche, du questionnaire et guide d'enquête, à l'élaboration de la partie théorique de la première version jusqu'à celle-ci.

\section{REMERCIEMENTS}

Nous remercions les producteurs du coton biologique et conventionnel des communes de Banikoara, Kandi, Glazoué et Tanguiéta, les secrétaires des coopératives, les agents d'encadrement des producteurs à la base qui de par leur franche collaboration ont contribué à la réussite de cette étude.

\section{REFERENCES}

Agbohessi TP, Toko II, Kestemont P. 2012. État des lieux de la contamination des écosystèmes aquatiques par les pesticides organochlorés dans le Bassin cotonnier béninois. Cahiers Agricultures, 21(2):
46-56.

DOI:

https://doi.org/10.1684/agr.2012.0535

Amonmidé I, Dagbenonbakin G, Agbangba CE, Akponikpe P. 2019. Contribution à l'évaluation du niveau de fertilité des sols dans les systèmes de culture à base du coton au Bénin. International Journal of Biological and Chemical Sciences, 13(3) : $\quad 1846-1860 . \quad$ DOI: https://dx.doi.org/10.4314/ijbcs.v13i3.52

Arrow K. 1969. Classificatory Notes on the Production and Transmission of Technological Knowledge, The American Economic Review. Papers and Proceedings, 59(2): 29-35. DOI: http://www.jstor.org Wed Nov 7 11:27:08 2007

Azonkpin S, Chougourou DC, Agbangba AC, Santos CCJ, Soumanou MM, Vodouhe SD. 2018. Typologie des système de culture de coton biologique au Bénin. International Journal of Biological and Chemical Sciences, 12(4): 1688-1704. DOI:

https://dx.doi.org/10.4314/ijbcs.v12i4.14

Bacyé B, Kambiré HS, Somé AS. 2019. Effets des pratiques paysannes de fertilisation sur les caractéristiques chimiques d'un sol ferrugineux tropical lessivé en zone cotonnière à l'Ouest du Burkina Faso. International Journal of Biological and Chemical Sciences, 13(6) : 2930-2941. DOI : https://dx.doi.org/10.4314/ijbcs.v13i6.39

Benham A, Benham L. 2005. Measuring the costs of exchange. Institutions, Contracts and Organisations: Perspectives from New Institutional Economics, Ménard C (ed.). Edward Elgar : Cheltenham, UK.

Bonou-zin RDC. 2012. Genre et performance économique des systèmes de production cotonnière du centre et au nord du Bénin. Mémoire de DEA, Université de Parakou, 93p.

Coase RH. 1937. The nature of the firm. Economica, 4(2): $\quad 386-405$. 
DOI: https://doi.org/10.1111/j.14680335.1937.tb00002.x

Deschesnes M, Couturier Y, Laberge S, Trudeau F, Bernier J, Manon B, Campeau L, Mababou K. 2008. Les facteurs influençant la dissémination et l'adoption de l'approche École en santé, Rapport de recherche.

http://www.fqrsc.gouv.qc.ca/fr/recherche -expertise/projets/rapports-

recherche.php\#habitudes, (19.11.19)

Degla KP. 2012, Transaction Costs in the Trading System of Cashew Nuts in the North of Benin: A Field Study. American Journal of Economics and Sociology, 71(2): 277-297. DOI : https://doi.org/10.1111/j.1536-

7150.2012.00830.x

Degla KP. 2017. Les Approches de l'Economie Néo-institutionnelle. Une Analyse Synthétique. Editions Universitaires Européennes.

De Soto H. 1990. The Other Path. New 10

B.C. Third World L.J. 204.

Dieye PN. 2006. Arrangements contractuels et performance des marchés du lait local au sud du Sénégal. Thèse de doctorat. Ecole Nationale Supérieure Agronomique de Montpellier. 211p.

Eggertson T. 1990. Economic Behavior and Institution, Cambridge.

Greene WH. 2008. Econometric Analysis, 6th ed. Pearson, Upper Saddle River NJ.

Gouda A-I, Imorou Toko I, Salami S-D, Richert M, Scippo M-L, Kestemont P, Schiffers B. 2018. Pratiques phytosanitaires et niveau d'exposition aux pesticides des producteurs de coton du nord du Bénin. Cah. Agric., 27(6): 19 .

DOI : https://doi.org/10.1051/cagri/2018038

Hougni A, Kpadé PC, Djihinto AC. 2012. Culture biologique du cotonnier et commerce équitable du coton : Deux approches de niche. Bull. Rech. Agron. Bénin, 229 (1) : 60-74.

Houndekon VA. 2014. Analyse comparative des systèmes de production du coton biologique et du coton conventionnel au Bénin. Journal de la Recherche Scientifique de l'Université de Lomé, 16(3): 8-21. DOI : https://www.ajol.info/index.php/jrsul/arti cle/view/117709

Hountondji SP. 2015. Analyse de l'efficacité économique de la production du coton biologique au Bénin. Mémoire de Master. Université de Parakou. 104p.

INSAE. 2019. Statistiques agricoles de la campagne agricole 2017-2018. 5p.

Klaes M. 2000. The birth of the concept of transaction costs: Issues and controversies. Industrial and Corporate Change, 9(4): 567-593. DOI: https://doi.org/10.1093/icc/9.4.567

North DC. 1987. Institutions, Transaction Costs and Economic Growth. Economic Inquiry 25(1): 419-428. DOI: https://doi.org/10.1111/j.14657295.1987.tb00750.x

Rogers EM. 1962. Diffusion of Innovations. The Free Press: New York; 236p.

Rogers EM. 1983. Diffusion of Innovations (Third Edition). The Free Press: New York; 290p.

Royer A. 2009. Analyse économique néoinstitutionnelle de la mise en marché collective. Application au secteur laitier du Québec. PhD Thesis. Université Panthéon-Sorbonne-Paris I. 350p.

Slangen LHG, Loucks AL, Slangen AHL. 2008. Institutional Economics and Economic Organisation Theory, an Integrated Approach. Academic ed.: Wageningen.

Sodjinou E, Glin LC, Nicolay G, Tovignan S, Hinvi J. 2015. Socioeconomic determinants of organic cotton adoption in Benin, West Africa. Agric. Food Econ., 12(3): 1-22. DOI: 10.1186/s40100-015-0030-9

Stiglitz JE. 1986. The New Development Economics. World Development, 14(2): 
257-265.

DOI :

https://doi.org/10.1016/0305-

750X(86)90057-4

Stiglitz JE. 1988. Economic Organization, Information, and Development. In Chenery, H. and T. Srinivasan. Handbook of Development Economics. T. Paul Schultz and John Strauss (ed). NorthHolland. 93-160.

Ton P. 2001. Cotton production and rural livelihoods in West Africa. Situation and trends in the context of trade liberalisation and structural adjustment. African Environment, Occasional Papers, Dakar (Senegal). Oxfam-GB \& ENDA-Tiers Monde. 87p.

Tovignan DS, Sodjinou E, Glin LC, Hinvi J, Bonou-zin DCR, Koussahoué S, Nicolay G. 2018. Analyse des déterminants de la rentabilité du coton biologique et conventionnel au Bénin. International Journal of Innovation and Scientific Research, 39 (1) : 79-90.

Vidjingninou F. 2018. Bénin : fort rebond de la production de coton en 2017-2018 https://www.jeuneafrique.com/575159/e conomie/benin-fort-rebond-de-laproduction-de-coton-en-20172018/(12.11.19)
Verbeek M. 2004. A Guide to Modern Econometrics (2nd edn). John Wiley \& Sons Ltd: West Sussex.

Williamson OE. 1990. A Comparison of Alternative Approaches to Economic Organisation. Journal of Institutional and Theoretical Economics, 146(1): 61-72.

Williamson OE. 1994. Les Institutions de l'Économie. Inter Editions : Paris ; 404p.

Williamson OE. 1998. Transaction Cost Economics. How it works, where it headed. De Economist, 146(1): 23-58. DOI :

https://doi.org/10.1023/A:100326390856 7

Williamson OE. 2000. The New Institutional Economics: Taking Stock, Looking Ahead. Journal of Economic Literature, 38 (3): 595-596.

Yabi JA, Bachabi FX, Labiyi IA, Ode CA, Ayena RL. 2016. Déterminants socioéconomiques de l'adoption des pratiques culturales de gestion de la fertilité des sols utilisées dans la commune de Ouaké au Nord-Ouest du Bénin. International Journal of Biological and Chemical Sciences, 10(2): 779-792. DOI: http://dx.doi.org/10.4314/ijbcs.v10i2.27. 